\title{
PRODUCCIÓN DE TOMATE EN INVERNADERO CON HUMUS DE LOMBRIZ COMO SUSTRATO
}

\author{
TOMATO PRODUCTION IN GREENHOUSE USING VERMICOMPOST AS SUBSTRATE
}

\author{
Norma Rodríguez Dimas ${ }^{1}$, Pedro Cano Ríos ${ }^{2 *}$, Uriel Figueroa Viramontes², Arturo Palomo Gil ${ }^{1}$, \\ Esteban Favela Chávez ${ }^{1}$, Vicente de Paul Álvarez Reyna1, Cándido Márquez Hernández ${ }^{3}$ y \\ Alejandro Moreno Reséndez ${ }^{4}$
}

\begin{abstract}
${ }^{1}$ Posgrado en Ciencias Agrarias, ${ }^{2}$ Departamento de Horticultura, y ${ }^{4}$ Departamento de Suelos, Universidad Autónoma Agraria Antonio Narro-Unidad Laguna. Periférico y Carretera Sta. Fe, s/n. Torreón, Coahuila. ${ }^{3}$ Escuela Superior de Biología, Universidad Juárez del Estado de Durango. Gómez Palacio, Durango.
\end{abstract}

*Autor para correspondencia (canorp49@hotmail.com)

\section{RESUMEN}

En condiciones de invernadero y durante el ciclo de invierno 2004 - 2005, se evaluaron dos híbridos de tomate (Lycopersicon esculentum Mill.) 'Big Beef' y 'Miramar' en tres sustratos: S1, vermicomposta + arena, en proporción 1:1 (v:v) + micronutrimentos quelatizados; S2, vermicomposta + arena, sin micronutrimentos; y el testigo S3, arena + solución nutritiva inorgánica. Los seis tratamientos se distribuyeron en un diseño completamente al azar con arreglo factorial $3 \times 2$ con 36 repeticiones, una planta por repetición. Se detectaron diferencias entre sustratos en rendimiento y calidad ( $P$ $\leq \mathbf{0 . 0 1}$ ). El híbrido 'Big Beef' en el $\mathrm{S3}$ presentó el rendimiento comercial más alto con $279 \mathrm{t} \mathrm{ha}^{-1}, \mathrm{y}$ superó al $\mathrm{S} 1$ con el mismo genotipo. En rendimiento de fruto total ambos híbridos crecidos en el S3 superaron al S1; sin embargo, el rendimiento en S1 fue mayor de $200 \mathrm{t} \mathrm{ha}^{-1}$ en ambos genotipos. Además, en S1 se logró mayor contenido de sólidos solubles y espesor del pericarpio en el fruto que en S3 y S2, por lo que el uso de vermicomposta + arena + quelatos puede ser una opción viable para producir tomate orgánico en invernadero.

Palabras clave: Lycopersicon esculentum, agricultura orgánica, cultivo protegido, vermicomposta.

\section{SUMMARY}

Two tomato hybrids (Lycopersicon esculentum Mill.) 'Big Beef' and 'Miramar', and tree substrates: S1, vermicompost + sand (1:1 in volume) + micronutrient chelates; $\mathbf{S 2}$, vermicompost + sand without chelates; and S3, sand + nutrient solution inorganic, were evaluated under greenhouse conditions during the 2004-2005 season. A $3 \times 2$ factorial arrangement was used. The six treatments were distributed in a completely randomized design with 36 replications, one plant per replication. Significant differences $(P \leq 0.01)$ were detected among substrates in fruit quality and yield. 'Big Beef' in the S3 substrate showed the highest commercial yield with 279 ha $^{-1}$, which was higher than in S1 substrate with the same hybrid. In total yield both cultivars grown in S3 yielded more than in S1; nonetheless, in S1 the fruit yield was higher than $200 \mathrm{t} \mathrm{ha}^{-1}$ with both hybrids. The soluble solids content and fruit thickness pericarp were higher in S1 than in S3 and S2. Therefore, the use of vermicompost
+ sand + quelates can be a viable option for producing organic tomato in greenhouse.

Index words: Lycopersicon esculentum, organic agriculture, vermicompost, protected crop.

\section{INTRODUCCIÓN}

La producción de tomate (Lycopersicon esculentum Mill.) en condiciones protegidas incrementa el rendimiento y calidad del fruto. La superficie empleada para cultivos en invernadero en México asciende a 4900 ha y presenta una tasa de crecimiento anual de $25 \%$; de esta superficie, 3450 ha se destinan a la producción de tomate (Fonseca, 2006). Los sistemas de producción varían en cuanto a variedades, sustratos de crecimiento, dosis de nutrimentos, técnicas de control de plagas y enfermedades, entre otros factores.

El uso excesivo de productos químicos en la agricultura preocupa a los consumidores por el nivel de contaminantes que los frutos pudiera contener, los problemas ambientales y la presencia de compuestos residuales en los suelos agrícolas (Eskenazi et al., 2004; Hernández et al., 2004). Para reducir el impacto de los agroquímicos sobre el ambiente y calidad de los productos vegetales y obtener productos inocuos, se recomiendan sistemas de producción orgánica que reduzcan o supriman el uso de fertilizantes, insecticidas, herbicidas, hormonas y reguladores de crecimiento inorgánicos (Ruiz, 1998; Milles y Peet, 2002; FAO, 2001b). Debido a la aceptación de los productos de este tipo, la superficie destinada a la agricultura orgánica ha registrado tasas de crecimiento mundiales superiores a $25 \%$ anual (Willer y Yussefi, 2000; Haring et al., 2001); además, los productos orgánicos tienen 
sobreprecios de 20 a $40 \%$ con respecto a los productos tradicionales (FAO, 2001a; Sloan, 2002).

Entre los abonos orgánicos de origen animal o vegetal (Tuzel et al., 2003; Rippy et al., 2004; Kamiar y Anusuya, 2005), la vermicomposta (VC) como sustrato permite satisfacer la demanda nutritiva de los cultivos en invernadero y reducir significativamente el uso de fertilizantes sintéticos (Manjarrez et al., 1999). El tomate 'Cherry' cultivado orgánicamente en invernadero produce un rendimiento de campo 16 veces superior que cuando se emplean sustratos de composta mezclada con medios inertes, con un promedio de $48.5 \mathrm{t} \mathrm{ha}^{-1}$ (Márquez et al., 2006).

Los beneficios de los abonos orgánicos son evidentes. La vermicomposta se utiliza como mejorador de suelo en cultivos hortícolas y como sustrato para cultivos en invernadero que no contamina el ambiente (Urrestarazu et al., 2001). La vermicomposta contiene sustancias activas que actúan como reguladores de crecimiento, elevan la capacidad de intercambio catiónico (CIC), tiene alto contenido de ácidos húmicos, y aumenta la capacidad de retención de humedad y la porosidad lo que facilita la aireación , drenaje del suelo y los medios de crecimiento (Ndegwa et al., 2000; Hashemimajd et al., 2004). Según Raviv et al. (2004, 2005), las compostas se usan como sustrato debido a su bajo costo, sustituyen al musgo y suprimen varias enfermedades presentes en el suelo. Moreno-Resendez et al. (2005) determinaron que la producción de tomate en invernadero puede efectuarse en mezclas de vermicomposta y arena como sustrato; ellos encontraron que 12.5 $\%$ de vermicomposta en mezclas con $87.5 \%$ de arena produjeron el mismo rendimiento $(\mathrm{P} \leq 0.05)$ que el testigo (arena con solución nutritiva), con 170 y $173.7 \mathrm{t} \mathrm{ha}^{-1}$ respectivamente.

La explotación intensiva de ganado lechero en la Comarca Lagunera, México genera una producción anual de estiércol bovino cercana a 900 mil toneladas (Figueroa, 2003). Este material puede emplearse para elaborar vermicomposta o humus de lombriz. En este estudio se postula que la vermicomposta derivada de estiércol de bovino puede usarse como fertilizante en la producción de tomate orgánico en invernadero. Por ello, el objetivo del presente trabajo fue evaluar el efecto de mezclas de vermicomposta de estiércol bovino en el rendimiento y calidad de fruto de dos híbridos comerciales de tomate, en condiciones de invernadero.

\section{MATERIALES Y MÉTODOS}

El experimento se hizo de octubre del 2004 a junio del 2005, en Torreón, Coahuila, México, en un invernadero de $200 \mathrm{~m}^{2}$, con cubierta de plástico, piso de grava y sis- tema de enfriamiento automático mediante pared húmeda y dos extractores. Se evaluaron tres sustratos en macetas de plástico de $18 \mathrm{~L}$ de capacidad: $\mathrm{S} 1$ = mezcla de arena + vermicomposta de estiércol bovino $(1: 1 \mathrm{v}: \mathrm{v})+$ micronutrimentos quelatos Maxiquel multi ${ }^{\circledR}(4 \% \mathrm{Fe}, 2 \% \mathrm{Zn}$, $1 \% \mathrm{Mn}) ; \mathrm{S} 2=$ arena + vermicomposta sin micronutrimentos; y S3 = arena + fertilizantes inorgánicos (testigo). Las macetas de los sustratos S2 y S3 se llenaron totalmente al momento del transplante, mientras que las macetas de S1 se llenaron con esta mezcla en forma gradual, $50 \%$ al transplante, $25 \%$ a los 79 d después de la siembra (dds), y $25 \%$ a los 134 dds. Antes de preparar las mezclas, la arena se lavó y esterilizó con una solución de hipoclorito de sodio a $5 \%$. Los micronutrimentos se suministraron a partir de los 74 dds hasta el final de la cosecha, en dosis de $1.15,0.49,0.16$ y $0.16 \mathrm{mg} \mathrm{L}^{-1} \mathrm{de}$ hierro, manganeso, zinc y boro, respectivamente. Se utilizaron dos híbridos de tomate, 'Big Beef' (BB) y 'Miramar' (M), ambos de la compañía Seminis Vegetable Seeds ${ }^{\circ}$, y se generaron seis tratamientos producto de un arreglo factorial $3 \times 2$, donde el factor A fueron los tres sustratos y el Factor B los dos genotipos, en un diseño completamente al azar con 36 repeticiones y una planta como unidad experimental.

La siembra fue el 2 de octubre de 2004, en charolas germinadoras de 200 cavidades llenas con "peat moss" como medio de crecimiento. El transplante se hizo el 20 de noviembre, y las macetas se acomodaron en doble hilera con una separación de $160 \mathrm{~cm}$ entre hileras y arreglo en "tresbolillo", con un espaciamiento de $30 \mathrm{~cm}$ de centro a centro de maceta, para una densidad de 4.2 plantas $\mathrm{m}^{-2}$. En el testigo se aplicó la solución nutritiva de Zaidan (1997) (Cuadro 1), con algunas modificaciones; además diariamente se aplicaron micronutrimentos quelatizados, por ser la arena un sustrato inerte, a través del sistema de riego por goteo. Según la etapa fenológica, en el agua de riego se aplicaron de 0.35 a $1.9 \mathrm{~L}$ por maceta. Las temperaturas máximas y mínimas medias dentro del invernadero fueron 12.4 y $32.9{ }^{\circ} \mathrm{C}$, respectivamente, durante el ciclo del cultivo que duró $215 \mathrm{~d}$.

Las plantas fueron guiadas a un solo tallo por eliminación de los brotes axilares, y tutoradas con rafia. Diariamente entre las 11 y $14 \mathrm{~h}$, se estimuló mecánicamente la polinización con un vibrador eléctrico. La cosecha de frutos se hizo del primero al octavo racimo para un total de 20 cortes, que iniciaron a los 139 dds.

La composición química de la vermicomposta se presenta en el Cuadro 2. El agua de riego tenía una conductividad eléctrica (CE) de $1.05 \mathrm{dS} \mathrm{m}^{-1}$, con una relación de absorción de sodio (RAS) de 2.18 y se clasificó como 
C1S1, de bajo riesgo de salinización y alcalinización (Cuadro 3) (Ayers y Westcot, 1994; Anónimo, 2004).

En cada planta se midió: rendimiento total (peso de todos los frutos contenidos en la planta $\mathrm{x}$ número de plantas por $\mathrm{m}^{2}$ ); número de frutos por planta; rendimiento comercial (la suma de rendimiento grande, mediano y chico); y rendimiento por tamaño, según la clasificación de la norma oficial NMX-FF-031-1997-SCFI (Cuadro 4). Los 20 cortes se hicieron entre el 8 de febrero y el 14 de junio de 2005. La calidad de fruto se basó en un muestreo de dos frutos de cada racimo en seis plantas; en los ocho racimos; los frutos con peso individual mayor de $100 \mathrm{~g}$ se seleccionaron de la parte media de cada racimo, y en cada fruto se evaluó: peso individual (g); diámetros polar y ecuatorial $(\mathrm{cm})$; contenido de sólidos solubles ( ${ }^{\circ}$ Brix); espesor de pulpa $(\mathrm{cm}) ; \mathrm{pH}$ del jugo; y número de lóculos por fruto. Los frutos se pesaron en una báscula digital (PS-5 Torrey). Los diámetros polar y ecuatorial se midieron con vernier $\left(\right.$ Scala $\left.{ }^{\circledR}\right)$. Los sólidos solubles se determinaron en una gota de jugo del fruto, con un refractómetro (ATARGO ATG-1E®). El espesor de la pulpa se midió en la parte carnosa del pericarpio del fruto con una regla $(\mathrm{cm})$. Los frutos con peso $<50 \mathrm{~g}$ o frutos dañados se clasificaron como rendimiento "rezaga". Se aplicó un análisis de varianza bajo el diseño completamente al azar, con el paquete estadístico Statistical Analysis System (SAS) versión 6.12 (SAS Institute, 1998) y se utilizó la prueba de Tukey $(\mathrm{P} \leq 0.05)$ para la comparación de medias.

\section{RESULTADOS Y DISCUSIÓN}

La interacción sustrato $\mathrm{x}$ genotipo fue significativa $(\mathrm{P}$ $\leq$ 0.01) para el rendimiento de los tres tamaños de fruto, grande, mediano y chico, así como para el peso de fruto, diámetro polar y número de lóculos (Cuadro 5). Para rendimiento rezaga, diámetro ecuatorial y espesor de pericarpio, sólo se detectaron diferencias entre genotipos, mientras que para el rendimiento total y comercial sólo hubo diferencias entre sustratos. Los sólidos solubles presentaron diferencias significativas para sustratos y genotipos, pero no para la interacción, y en el pH no se detectaron diferencias para sustrato, genotipo ni sustrato $\mathrm{x}$ genotipo.

\section{Rendimiento total de fruto}

El híbrido 'Big Beef' en el sustrato S3 presentó el mayor rendimiento con $279.3 \mathrm{t} \mathrm{ha}^{-1}$, seguido de 'Miramar' en el mismo sustrato (Cuadro 6). El rendimiento total (RT) de ambos híbridos fue estadísticamente igual en el sustrato S1. Los rendimientos más bajos de ambos híbridos se obtuvieron con el sustrato S2, con valores entre 139 y $156 \mathrm{t} \mathrm{ha}^{-1}$ (Figura 1). El híbrido 'Big Beef' redujo más su rendimiento que 'Miramar' al cambiar del sustrato de arena con solución nutritiva S3, a S1 y a S2 (Cuadro $6)$.

Cuadro 1. Concentración de la solución nutritiva (ppm) según la etapa fenológica (mg g ${ }^{-1}$ ) (Zaidan, 1997). Torreón, Coah. 2005.

\begin{tabular}{lcccc}
\hline & \multicolumn{3}{c}{ Elemento nutrimental } \\
\cline { 2 - 5 } Etapa fenológica & $\mathrm{N}$ & $\mathrm{P}$ & $\mathrm{K}$ & $\mathrm{Ca}$ \\
\hline Plantación y establecimiento & $100-120$ & $40-50$ & $150-160$ & $100-120$ \\
Floración y cuajado & $150-180$ & $40-50$ & $200-220$ & $100-120$ \\
Inicio de maduración y cosecha & $180-200$ & $40-50$ & $230-250$ & $100-120$ \\
\hline
\end{tabular}

Cuadro 2. Análisis químico de la vermicomposta y arena de los sustratos. Torreón, Coah. 2005.

\begin{tabular}{|c|c|c|c|c|c|c|c|c|c|c|c|}
\hline \multirow{2}{*}{ 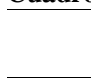 } & \multirow{2}{*}{$\begin{array}{l}\mathrm{N} \\
(\%)\end{array}$} & \multirow[t]{2}{*}{$\mathrm{P}$} & \multirow[t]{2}{*}{$\mathrm{K}$} & \multirow[t]{2}{*}{$\mathrm{Ca}$} & \multirow[t]{2}{*}{$\mathrm{Mg}$} & \multirow[t]{2}{*}{$\mathrm{Na}$} & $\mathrm{Fe}$ & $\mathrm{Zn}$ & $\mathrm{Mn}$ & \multirow[t]{2}{*}{$\mathrm{pH}$} & \multirow{2}{*}{ 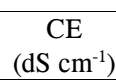 } \\
\hline & & & & & & & \multicolumn{3}{|c|}{$\left(\mathrm{mg} \mathrm{kg}^{-1}\right)$} & & \\
\hline $\mathrm{VC}$ & 0.97 & 0.199 & 1.03 & 4.86 & 0.76 & 0.14 & 3.9 & 1.45 & 3.71 & 8.2 & 2.4 \\
\hline A & 0.011 & 0.0005 & 0.01 & 0.004 & 0.0016 & 0.007 & 2.1 & 0.90 & 1.65 & 7.5 & 0.65 \\
\hline
\end{tabular}

$\mathrm{VC}=$ vermicomposta; $\mathrm{A}=$ arena.

Cuadro 3. Análisis químico del agua de riego utilizada en el experimento. Torreón, Coah. 2005

\begin{tabular}{|c|c|c|c|c|c|c|c|c|c|}
\hline & $\mathrm{CE}$ & $\mathrm{pH}$ & $\mathrm{K}^{+}$ & $\mathrm{Ca}^{+}$ & $\mathrm{Mg}^{+}$ & $\mathrm{Na}^{+}$ & $\mathrm{HCO}_{3}^{-}$ & $\mathrm{Cl}^{-}$ & $\mathrm{SO}_{4}{ }^{-2}$ \\
\hline & $\left(\mathrm{dS} \mathrm{cm} \mathrm{cm}^{-1}\right)$ & & \multicolumn{7}{|c|}{$\left(\right.$ meq L $\left.\mathrm{L}^{-1}\right)$} \\
\hline Contenido & 1.05 & 8.75 & 1.4 & 4.7 & 0.80 & 3.63 & 0.55 & 2.3 & 4.1 \\
\hline
\end{tabular}


Dado que el testigo S3 superó a los dos sustratos, se infiere que la vermicomposta en el sistema de producción S2 no cubrió las necesidades nutricionales del cultivo, lo que coincide con Betiol et al. (2004), Márquez y Cano (2004) y Heeb et al. (2005), quienes encontraron mayor rendimiento en los sistemas con fertilización inorgánica que en el sistema orgánico. Al respecto, Atiyeh et al. (2000) señalaron que el uso de más de $20 \%$ de vermicomposta en el sustrato hace disminuir la producción debido a un incremento en la conductividad eléctrica.

Cuadro 4. Clasificación del fruto por tamaño en tomate tipo bola (norma NMX-FF-031-1997-SCFI).

\begin{tabular}{lcc}
\hline \multirow{2}{*}{ Tamaño } & \multicolumn{2}{c}{ Diámetro } \\
\cline { 2 - 3 } & $\begin{array}{c}\text { Mínimo }^{\dagger} \\
(\mathrm{mm})\end{array}$ & $\begin{array}{c}\text { Máximo }^{\ddagger} \\
(\mathrm{mm})\end{array}$ \\
\hline Chico & 54 & 57 \\
Mediano & 58 & 63 \\
Grande & 64 & 70 \\
Extragrande & 71 & $>71$ \\
\hline
\end{tabular}

${ }^{\dagger}$ En posición vertical, el fruto no puede pasar por una abertura circular del diámetro designado.

*En cualquier posición, el fruto puede pasar por una abertura circular del diámetro designado.
El mayor rendimiento total de fruto de $\mathrm{S} 1$ comparado con S2 en los dos híbridos, pudo deberse a que la aplicación gradual de vermicomposta a los 79 y 134 dds coincidió con las etapas de floración y desarrollo del fruto, cuando la planta requiere mayor cantidad de nutrimentos. En los sustratos S1 y S2 se presentaron síntomas visuales de deficiencia de $\mathrm{N}$ durante floración aunque con los aportes fraccionados de vermicomposta en $\mathrm{S} 1$ se controló esta deficiencia. El agregar la mezcla orgánica en el sustrato S2 en una sola aplicación durante el trasplante, pudo inducir una deficiencia de $\mathrm{N}$ por lixiviación, con la consecuente reducción en la producción. De acuerdo con datos del Servicio de Conservación de Recursos Naturales de Estados Unidos (NRCS, 1999) y de Castilla (2003), una cosecha de tomate extrae $3 \mathrm{~kg}$ de nitrógeno por tonelada de fruto en fresco; en el presente estudio se supuso una extracción similar, de modo que la variedad 'Big Beef' extrajo el equivalente a $20.1 \mathrm{~g}$ por planta, para un rendimiento de $6.71 \mathrm{~kg}$ de tomate por planta.

Cuadro 5. Cuadrados medios de los análisis de varianza para variables de rendimiento, tamaño y calidad de frutos de dos híbridos de tomate bajo invernadero en tres sustratos. Torreón, Coah. 2005.

\begin{tabular}{|c|c|c|c|c|c|}
\hline Característica & Sustrato (S) & Genotipos (G) & $\mathrm{S} \times \mathrm{G}$ & Error & $\mathrm{CV}(\%)$ \\
\hline $\mathrm{R}$ total & $221920 * *$ & $3644 \mathrm{~ns}$ & $15745 \mathrm{~ns}$ & 6213 & 38 \\
\hline $\mathrm{R}$ grande & $230070 * *$ & $623563 * *$ & $41556^{* *}$ & 3917 & 54 \\
\hline $\mathrm{R}$ medio & $3132 *$ & 180170 ** & $12893 * *$ & 1400 & 65 \\
\hline $\mathrm{R}$ chico & $6831 * *$ & $85568 * *$ & $4100 * *$ & 294 & 59 \\
\hline R rezaga & $137 \mathrm{~ns}$ & $196^{* *}$ & $50 \mathrm{~ns}$ & 4596 & 67 \\
\hline R comercial & $218637^{* *}$ & $5281 \mathrm{~ns}$ & $16665 \mathrm{~ns}$ & 6299 & 39 \\
\hline Número de fruto & $2363^{* *}$ & $17103 * *$ & $121 \mathrm{~ns}$ & 131 & 32 \\
\hline Peso de fruto & $7003 *$ & $152567 * *$ & $12012 * *$ & 15467 & 22 \\
\hline Diámetro ecuatorial & $0.48 \mathrm{~ns}$ & $37.4 * *$ & $1.04 \mathrm{~ns}$ & 0.52 & 8 \\
\hline Diámetro polar & $1.48^{* *}$ & $22.8^{* *}$ & $1.03 * *$ & 0.13 & 15 \\
\hline No. de lóculos & $1.38 \mathrm{~ns}$ & $311.5 * *$ & $3.94 * *$ & 0.76 & 21 \\
\hline Sólidos solubles & $5.72 * *$ & $1.2^{*}$ & $0.66 \mathrm{~ns}$ & 0.24 & 9 \\
\hline Espesor pericarpio & $0.02 \mathrm{~ns}$ & $0.98 * *$ & $0.01 \mathrm{~ns}$ & 0.007 & 11 \\
\hline $\mathrm{pH}$ & $0.02 \mathrm{~ns}$ & $0.001 \mathrm{~ns}$ & $0.014 \mathrm{~ns}$ & 0.024 & 4 \\
\hline
\end{tabular}

$\mathrm{ns}, * \mathrm{y} * *=$ No significativo, significativo con $\mathrm{P}<0.05$ y $\mathrm{P}<0.01$, respectivamente; $\mathrm{R}=$ rendimiento; $\mathrm{C} \mathrm{V}=$ Coeficiente de variación.

Cuadro 6. Rendimiento de frutos como suma de 20 cortes, en tres sustratos y tres genotipos, sobre la base de diámetros de fruto de tomate en 2004 2005 en la Comarca Lagunera. Torreón, Coah.

\begin{tabular}{|c|c|c|c|c|c|c|}
\hline Tratamientos & $\begin{array}{l}\text { Rendimiento T } \\
\left(\mathrm{t} \mathrm{ha}^{-1}\right)\end{array}$ & Grande & Mediano & Chico & Rezaga & Comercial \\
\hline S2 'Big Beef' & $139.7 \mathrm{c}$ & $92.6 \mathrm{c}$ & $35.7 \mathrm{c}$ & $10.7 \mathrm{~d}$ & 0.72 & $139.0 \mathrm{c}$ \\
\hline S3 'Big Beef' & $279.3 \mathrm{a}$ & $251.6 \mathrm{a}$ & $21.0 \mathrm{c}$ & $6.4 \mathrm{~d}$ & 0.85 & $278.9 \mathrm{a}$ \\
\hline S1 'Miramar' & $208.3 \mathrm{~b}$ & $48.0 \mathrm{~d}$ & $92.5 \mathrm{a}$ & $64.1 \mathrm{a}$ & 3.47 & $204.5 \mathrm{~b}$ \\
\hline S2 'Miramar' & $156.1 \mathrm{c}$ & $37.7 \mathrm{~d}$ & $64.8 \mathrm{~b}$ & $52.8 \mathrm{~b}$ & 3.52 & $155.2 \mathrm{c}$ \\
\hline S3 'Miramar' & $238.3 \mathrm{~b}$ & $102.5 \mathrm{c}$ & $102.9 \mathrm{a}$ & $30.2 \mathrm{c}$ & 2.71 & $235.6 \mathrm{~b}$ \\
\hline Media general & 205.0 & 116.4 & 57.8 & 29.1 & 2.1 & 203.4 \\
\hline
\end{tabular}

Genotipos con letras iguales en una columna no son estadísticamente diferentes (Tukey, 0.05). ns, * y ** = No significativo, significativo a P $\leq 0.05$, y significativo a $\mathrm{P}<0.01 . \mathrm{T}=$ total; $\mathrm{S} 1=$ mezcla de arena + vermicomposta gradual $(1: 1 \mathrm{v}: \mathrm{v})+$ quelatos; $\mathrm{S} 2=$ arena + vermicomposta sin micronutrimentos; y S3 = arena + fertilizantes inorgánicos (testigo). 


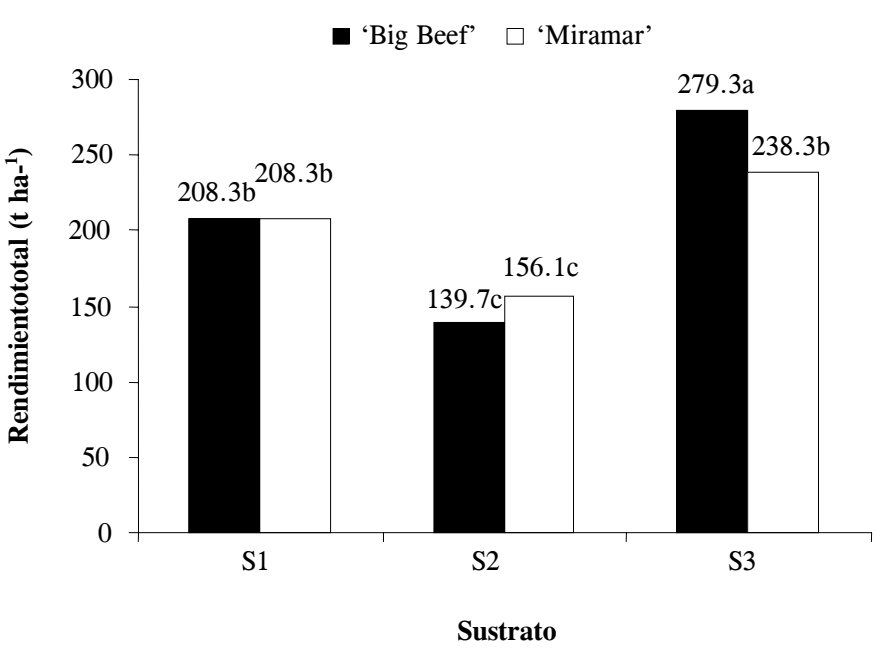

Figura 1. Rendimiento total de híbridos de tomate cultivados en tres sistemas de fertilizante en invernadero. Comarca Lagunera, 20042005. S1 = vermicomposta + arena, en proporción 1:1 (v:v) + micronutrimentos quelatizados; S2 $=$ vermicomposta + arena 1:1 (v:v), sin micronutrimentos; y S3 = arena + fertilizante inorgánico.

En la solución nutritiva utilizada se aportó un total de $21.5 \mathrm{~g}$ de $\mathrm{N}$ por maceta en el ciclo, con un volumen de riego promedio de $1.46 \mathrm{~L} \mathrm{~d}^{-1}$ por maceta. Sin embargo, Castellanos (2004) comenta que del sustrato se lixivia hasta $42 \%$ del $\mathrm{N}$ en condiciones de invernadero.

En el sustrato orgánico $\mathrm{S} 1$ y según el análisis de la vermicomposta, la aportación de $\mathrm{N}$ total fue de $65.5 \mathrm{~g}$ por maceta, mientras que el requerimiento de $\mathrm{N}$ promedio de los dos genotipos fue de $14.7 \mathrm{~g}$ por maceta, para un rendimiento de $4.9 \mathrm{~kg}$ de tomate por planta. La mayor parte del $\mathrm{N}$ en las compostas es orgánico y durante el proceso de mineralización se libera el $\mathrm{N}$ inorgánico disponible al cultivo. Eghball (2000) encontró una tasa de mineralización de $\mathrm{N}$ en composta de bovino de $11 \%$; si se supone que una tasa similar en la vermicomposta, la liberación de $\mathrm{N}$ en el presente estudio habría sido de $7.2 \mathrm{~g}$ por maceta. Como la mineralización de $\mathrm{N}$ se incrementa con la temperatura (Griffin y Honeycutt, 2000), sería de esperarse una mayor tasa de mineralización de $\mathrm{N}$ en las condiciones del presente estudio. Aun así, se detectaron síntomas de deficiencia de $\mathrm{N}$ a partir de la etapa de floración. Lo anterior concuerda con Raviv et al. (2004), quienes concluyeron que después de dos meses del trasplante con vermicomposta se requiere fertilización, ya que los nutrimentos se lixivian o se absorben por la planta.

La disminución en la producción de S1 podría ser compensada con el valor del producto. Según Gómez et al. (2003) y Sloan (2002) el precio del producto orgánico se incrementa de 20 a $40 \%$ con respecto al del sistema tradicional. Así mismo, el sustrato S1 podría recomendar- se si se alterna con aplicaciones de fertilizantes orgánicos o té de composta, como sugirieron Cano et al. (2005).

Si se considera que una producción comercial exitosa de tomate en invernadero es de $200 \mathrm{t} \mathrm{ha}^{-1}$ por año como mínimo (Cotter y Gómez, 1981), entonces solamente el sustrato $\mathrm{S} 1$ es viable, ya que presentó un rendimiento de $208 \mathrm{t} \mathrm{ha}^{-1}$ en un periodo de nueve meses. Entre los dos sustratos orgánicos se encontró que el S1 rindió más que el S2, lo que se atribuye tanto a la aplicación dividida de la vermicomposta como a la aplicación de los micronutrimentos añadidos solamente a $\mathrm{S} 1$; estas sustancias no cancelan la clasificación del sustrato como orgánico ya que los micronutrimentos (oligoelementos; boro, cobre, hierro, magnesio, molibdeno, zinc) son sustancias que pueden emplearse como fertilizantes y acondicionadores del suelo en producción orgánica (FIRA, 2003; FAO, 2001b).

\section{Rendimiento por tamaño de fruto}

En el fruto de tamaño grande, los rendimientos más altos se presentaron en el sustrato S3 y el híbrido 'Big Beef' con $251.6 \mathrm{t} \mathrm{ha}^{-1}$, en contraste con el sustrato S2 combinado con el mismo genotipo que dio un rendimiento de $92.6 \mathrm{t} \mathrm{ha}^{-1}$ (Cuadro 6). El rendimiento medio de fruto grande de este híbrido en el sustrato S1 $\left(166.4 \mathrm{t} \mathrm{ha}^{-1}\right)$ superó el de $40 \mathrm{t} \mathrm{ha}^{-1}$ registrado por Muro et al. (2003) y el de $101 \mathrm{t} \mathrm{ha}^{-1}$ por Ortega et al. (2003).

En la categoría de tamaño mediano el híbrido 'Miramar' presentó los mayores rendimientos en los sustratos S3 y S1, con 103 y 92.5 t ha $^{-1}$ respectivamente. En este tamaño 'Miramar' fue mejor que 'Big Beef' en los tres sustratos (Cuadro 6), debido a que este último genotipo produce frutos grandes. El sustrato $\mathrm{S} 2$ con un rendimiento de $64.8 \mathrm{t} \mathrm{ha}^{-1}$ superó a los obtenidos por Muro et al. (2003) y Ortega et al. (2003), que fueron de 26 y 23 t ha 1 . Para el fruto chico, las combinaciones de los sustratos S1 y S2 con el genotipo 'Miramar', presentaron el mayor rendimiento, con 64 y $53 \mathrm{t} \mathrm{ha}^{-1}$ respectivamente.

En la categoría rezaga no hubo diferencia significativa entre sustratos ni en la interacción sustrato $\mathrm{x}$ genotipo (Cuadro 5), pero la producción promedio de 'Miramar' de $3.2 \mathrm{t} \mathrm{ha}^{-1}$ superó a 'Big Beef' que rindió $0.92 \mathrm{t} \mathrm{ha}^{-1}$. Entonces, este último híbrido resulta un buen candidato para la producción de fruto comercial porque alcanzó el mayor rendimiento $\left(278.9 \mathrm{t} \mathrm{ha}^{-1}\right)$ en esta categoría.

Los resultados de las mezclas de vermicomposta + arena (S1) concuerdan con los de Atiyeh et al. (2000), quienes encontraron que las diferencias entre sustratos se deben a diferencias en sus contenidos de elementos 
nutritivos y a la naturaleza de sus comunidades microbianas. Los sustratos S3 y S1 presentaron mayor cantidad de frutos por planta, y fueron estadísticamente iguales entre sí, con 40 y 37 frutos respectivamente, mientras que S2 dio el menor valor con 29 frutos.

Los coeficientes de correlación (Cuadro 7) muestran las variables que más se asocian con el rendimiento total son: rendimiento de fruto grande $\left(\mathrm{r}=0.73^{* *}\right)$; número de frutos por planta $\left(\mathrm{r}=0.69^{* *}\right)$; y rendimiento de fruto mediano $\left(\mathrm{r}=0.46^{* *}\right)$. El rendimiento rezaga $(\mathrm{RR})$, chico $(\mathrm{RCH})$ y peso de fruto $(\mathrm{PF})$ no correlacionaron con el rendimiento total.

\section{Calidad de fruto}

La combinación de los sustratos S3 y S1 con el híbrido 'Big Beef' presentó el mayor peso de fruto (214 y 209 g, respectivamente) y mayor diámetro polar $(6.3$ y $6.1 \mathrm{~cm}$, respectivamente) que fueron iguales entre sí y superaron al híbrido 'Miramar' en los tres sustratos, ya que sus promedios respectivos fueron $147 \mathrm{~g}$ y $5.4 \mathrm{~cm}$, (Cuadro 8). Resultados semejantes encontraron Logendra et al. (2001) para peso de fruto, y Ortega et al. (2003) para diámetro polar. 'Big Beef' también presentó el mayor diámetro ecuatorial con $7.8 \mathrm{~cm}$ (Cuadro 8), lo que concuerda con lo obtenido por Márquez y Cano (2004) en este mismo genotipo.

'Miramar' mostró el mayor espesor de pericarpio en todos los sustratos, con una media de $0.84 \mathrm{~cm}$, en contraste con 'Big Beef' que tuvo $0.70 \mathrm{~cm}$ (Cuadro 8). Estos resultados no coinciden con los de Márquez y Cano (2004), quienes reportaron un espesor de $0.73 \mathrm{~cm}$ en la pulpa del hibrído 'Big Beef'. El pH promedio de fruto fue 4.2 (Cuadro 8), que es inferior al reportado por Bugarin et al. (2002) e Hidalgo et al. (1998). 'Big Beef' produjo menos frutos por planta, pero más grandes y de menor espesor de pericarpio. Por su parte, 'Miramar' produjo más frutos por planta, con mayor número de frutos medianos y pequeños y de mayor espesor de pericarpio (Cuadro 8).

Cuadro 7. Correlaciones entre variables de rendimiento en genotipos de tomate crecidos en sustratos bajo condiciones de invernadero en 2004-2005 en la Comarca Lagunera. Torreón, Coah.

\begin{tabular}{|c|c|c|c|c|c|c|c|}
\hline & R Total & R Grande & R Mediano & R Chico & R Rezaga & NF & PF \\
\hline R Grande & & & $-0.270 * *$ & $-0.516 * *$ & $-0.280 * *$ & $0.059 \mathrm{~ns}$ & $0.224 * *$ \\
\hline R Mediano & & & & $0.457 * *$ & $0.064 \mathrm{~ns}$ & $0.775 * *$ & $-0.259 * *$ \\
\hline R Chico & & & & & $0.332 * *$ & $0.621 * *$ & $-0.253 * *$ \\
\hline NF & & & & & & & $-0.152 *$ \\
\hline
\end{tabular}

$\mathrm{ns}, * \mathrm{y} * *=$ No significativo, significativo con $\mathrm{P} \leq 0.05 \mathrm{y} \mathrm{P} \leq 0.01$, respectivamente. $\mathrm{R}=$ rendimiento; $\mathrm{NF}=$ número de frutos por planta; $\mathrm{y}$ PF $=$ peso de fruto.

\begin{tabular}{|c|c|c|c|c|c|c|c|}
\hline Sustrato & Genotipo & $\begin{array}{l}\text { Peso } \\
(\mathrm{g})\end{array}$ & $\begin{array}{l}\text { Diámetro } \\
\text { polar } \\
(\mathrm{cm})\end{array}$ & $\begin{array}{l}\text { Diámetro } \\
\text { ecuatorial } \\
(\mathrm{cm})\end{array}$ & $\begin{array}{l}\text { Espesor } \\
\text { pericarpio } \\
(\mathrm{cm})\end{array}$ & $\begin{array}{l}\mathrm{pH} \text { del } \\
\text { fruto }\end{array}$ & $\begin{array}{l}\text { Número } \\
\text { de frutos } \\
\text { por planta }\end{array}$ \\
\hline$\overline{S 1}$ & 'Big Beef' & $209 a$ & $6.1 \mathrm{a}$ & 7.8 & 0.73 & 4.3 & 27 \\
\hline S2 & 'Big Beef' & $173 \mathrm{~b}$ & $5.8 \mathrm{ab}$ & 7.5 & 0.72 & 4.2 & 22 \\
\hline S3 & 'Big Beef' & $214 \mathrm{a}$ & $6.3 \mathrm{a}$ & 8.0 & 0.68 & 4.2 & 32 \\
\hline Media 'Big Beef' & & 199 a & $6.1 \mathrm{a}$ & $7.8 \mathrm{a}$ & $0.70 \mathrm{~b}$ & 4.2 & $27 \mathrm{~b}$ \\
\hline $\mathrm{S} 1$ & 'Miramar' & $144 \mathrm{c}$ & $5.4 \mathrm{~b}$ & 7.0 & 0.85 & 4.2 & 47 \\
\hline $\mathrm{S} 2$ & 'Miramar' & $150 \mathrm{bc}$ & $5.5 \mathrm{~b}$ & 6.9 & 0.84 & 4.2 & 37 \\
\hline S3 & 'Miramar' & $145 \mathrm{c}$ & $5.5 \mathrm{~b}$ & 6.9 & 0.83 & 4.2 & 48 \\
\hline Media 'Miramar' & & $147 \mathrm{~b}$ & $5.4 \mathrm{~b}$ & $6.9 \mathrm{~b}$ & $0.84 \mathrm{a}$ & 4.2 & $44 \mathrm{a}$ \\
\hline $\mathrm{CV}(\%)$ & & 22 & 15 & 8 & 11 & 4 & 32 \\
\hline Tukey & & 10.3 & 0.093 & 0.19 & 0.022 & 0.57 & 2.9 \\
\hline Media general & & 171.5 & 5.8 & 7.3 & 0.78 & 4.2 & 36 \\
\hline
\end{tabular}

Medias con misma letra dentro de columnas son estadísticamente iguales (Tukey, 0.05). 
En sólidos solubles, en promedio de ambos híbridos, los sustratos S1, S2 y S3 presentaron en promedio 5.3, 5.1 y $4.9{ }^{\circ}$ Brix respectivamente. En el sustrato $\mathrm{S} 1$ se registró $8 \%$ más de sólidos solubles que en el testigo S3 (Cuadro 9). El genotipo 'Big Beef' con $5.1^{\circ}$ Brix, superó a 'Miramar' que presentó $4.9^{\circ} \mathrm{Brix}$, en promedio en los tres sustratos (Cuadro 9). En este experimento los genotipos presentaron buena calidad, ya que el tomate para procesado y consumo en fresco deben tener un contenido de sólidos solubles de 4.5 a $5.5{ }^{\circ}$ Brix (Díez, 1999). Frutos en este rango de sólidos solubles también lo obtuvieron Márquez y Cano (2004), Ortega et al. (2003) y MorenoReséndez et al. (2005).

Cuadro 9. Contenido de sólidos solubles de dos híbridos de tomate cultivados en tres sustratos en 2004-2005 en la Comarca Lagunera.

\begin{tabular}{lccc}
\hline Sustrato & 'Big Beef' & 'Miramar' & Media \\
\hline S1 & 5.3 & 5.3 & $5.3 \mathrm{a}$ \\
S2 & 5.3 & 5.1 & $5.1 \mathrm{~b}$ \\
S3 & 4.8 & 5.0 & $4.9 \mathrm{~b}$ \\
Media & $5.1 \mathrm{a}$ & $4.9 \mathrm{~b}$ & 5.1 \\
\hline
\end{tabular}

Medias con diferente letra son estadísticamente diferentes (Tukey, 0.05).

Valor de Tukey, 0.05 en sustrato $=0.252$ y en genotipo $=0.129$

\section{CONCLUSIONES}

El sustrato con fertilizantes químicos (S3) superó a los sustratos con vermicomposta S1 y a S2 en rendimiento total, comercial y calidad de fruto. El híbrido 'Big Beef' en el sustrato $\mathrm{S} 3$ presentó el más alto rendimiento comercial con 279 t ha-1. Las mezclas orgánicas S1 y S2 no satisfacieron las necesidades nutricionales de estos dos híbridos de tomate bajo las condiciones en que se realizó la evaluación. No obstante, es posible que el sustrato S1 pueda utilizarse en la producción de tomate orgánico en invernadero con cualquiera de los híbridos evaluados, ya que la disminución en producción se podría compensar con el valor comercial del producto y con el menor costo de fertilización.

\section{AGRADECIMIENTOS}

A la Fundación Produce Coahuila y Durango, por el apoyo económico. Al Departamento de Horticultura de la Universidad Autónoma Agraria Antonio Narro-Unidad Laguna, por las facilidades de infraestructura otorgadas al proyecto. Al Dr. Jesús Vázquez Arroyo, a la Q.F.B. Norma Lidia Rangel Carrillo y a la I.I.Q. Elba Margarita Aguilar Medrano, y a los ingenieros Eduardo Lara, Rey Idael Partida Morales y Luis Alberto Ramírez Estrada, por su colaboración en el desarrollo del experimento.

\section{BIBLIOGRAFÍA}

Anónimo (2004) Diagnóstico de aguas de riego. Disponible en: http://www.infoagro.com/riegos/diagnostico_aguas.htm. (consulta 15 de agosto 2006).

Atiyeh R M, N Arancon, C A Edwards, J D Metzger (2000) Influence of earthworm-processed pig manure on the growth and yield of greenhouse tomatoes. Bioresource Technol. 75:175180.

Ayers RS, DW Westcot (1994) Water Quality for Agriculture. FAO Irrigation and Drainage Paper 29 Rev. 1. FAO. Rome. 174 p.

Bettiol W, R Ghini, J A G Haddad, R S Cássio (2004) Organic and conventional tomato cropping systems. Sci. Agric. 61:253259.

Bugarin M R, A Galvis-Spinola, P Sánchez-García, D GarcíaParedes (2002) Demanda de potasio del tomate tipo saladette. Terra 20:391-399.

Castellanos J Z (2004) Manejo de la fertirrigación en suelo. In: Manual de Producción Hortícola en Invernadero. J Z Castellanos R, J J Muñoz (eds). 2a ed. Ed. Intagri. INCAPA. pp:103-123.

Castilla N (2003) Estructuras y equipamientos de invernaderos. In: Memoria del Curso Internacional de Producción de Hortalizas en Invernadero. J Z Castellanos R, J J Muñoz (eds). INIFAP. México. pp:1-11.

Cano R P, C Márquez H, U Figueroa V, N Rodríguez D, V Martínez C, A Moreno R (2005) Producción orgánica de tomate bajo invernadero en la Comarca Lagunera. In: Memoria de la XVII Semana Internacional de Agronomía, FAZ-UJED. J J Martínez R, S Berúmen P, J Martínez T, A Martínez R, M Vázquez N (eds). Gómez Palacio, Dgo. 5-9 Sep. pp:30-54.

Cotter D J, R E Gómez (1981) Cooperative Extension Service. 400 H11. U. New Mexico, USA. 4 p.

Diez J M (2001) Tipos varietales. In: El Cultivo del Tomate. Nuez F. (ed). Ed. Mundi-Prensa México. pp:95-129.

Eghball B (2000) Nitrogen mineralization from field-applied beef cattle feedlot manure or compost. Soil Sci. Soc. Amer. J. 64:20242030.

Eskenazi B, K Harley, A Bradman, E Weltzien, N P Jewell, D B Barr, C E Furlong, N T Holland (2004) Association of in utero organophosphate pesticide exposure and fetal growth and length of gestation in an agricultural population. Environ. Health Persp. 112:1116-1124.

FAO (2001a) Centro de Comercio Internacional. Los mercados mundiales de frutas y verduras orgánicas: oportunidades para los países en desarrollo en cuanto a la producción y exportación de productos hortícolas orgánicos. Roma, Italia. Disponible en: www.fao.org/docrep/004/y1669e/y1669e00.htm. (consulta 15 de noviembre del 2005).

FAO (2001b) La comisión del Codex Alimentarius y el programa conjunto FAO/OMS sobre normas alimentarias. SIN 1020-2579. 00100 Roma Italy ANEXO 2: Substancias permitidas para la producción de alimentos orgánicos. pp:54-60. Disponible en: http://www.fao.org/DOCREP/005/Y2772S/y2772s0c.htm (consulta 15 de noviembre del 2005).

FIRA, Fideicomisos Instituidos en Relación con la Agricultura (2003) Agricultura Orgánica. Normas internacionales ANEXOS. Apéndice 1, Productos Permitidos. A. Fertilizantes del Suelo y Vegetales. Boletín Informativo. Núm. 322 Volumen XXXV 10a. Epoca Año XXXI Diciembre 2003. pp:105-106.

Figueroa V U (2003) Uso sustentable del suelo. In: Abonos Orgánicos y Plasticultura. Gómez Palacio, Durango, México. FAZ UJED. SMCS y COCYTED. pp:1-22.

Fonseca A E (2006) Producción de tomate en invernadero. In: Cuarto Simposio Internacional de Producción de cultivos en Invernadero. E Olivares S (ed). UANL. Facultad de Agronomía. Monterrey, N. L. México. pp:1-8. 
Griffin T S, C W Honeycutt (2000) Using growing degree days to predict nitrogen availability from livestock manures. Soil Sci. Soc. Amer. J. 64:1876-1882.

Gómez C MA, L T Gómez, R Schwentesius (2003) Agricultura orgánica de México. In: Producción, Comercialización y Certificación de la Agricultura Orgánica en América Latina. CIESTAAM-AUNA, Edo. de México. pp:91-108.

Haring A, S Dabbert, F Offerman, H Nieberg (2001) Benefits of organic farming to society. In: Danish Ministry of Food and Fisheries. Organic Food and Farming: Towards Partnership and Action in Europe. Proceedings, $10-11$ de Mayo. 80 p.

Hashemimajd K, M Kalbasi, A Golchin, H Shariatmandari (2004) Comparison of vermicompost and compost as potting media for growth of tomatoes. J. Plant Nutr. 27:1107-1123.

Heeb A, B Lundegardh, T Ericsson, G P Savage (2005) Nitrogen form affects yield taste of tomatoes. J. Sci. Food Agric. 85:1405-1414

Hernández A, A Gomez M, G Pena, F Gil, L Rodrigo, E Villanueva, A Pla (2004) Effect of long-term exposure to pesticides on plasma esterases from plastic greenhouse workers. J. Toxicol. Environ. Health. Part A. 67:1095-1108.

Hidalgo G J C, G Alcántar G, G A Baca C, P Sánchez G, J A Escalante E (1998) Efecto de la condición nutrimental de las plantas y de la composición, concentración y $\mathrm{pH}$ del fertilizante foliar, sobre el rendimiento y calidad del tomate. Terra 16:143-148

Kamiar A, R Anusuya (2005) Compost for nitrogen fertility management of bell pepper in a drip-irrigated plasticulture system. HortScience 4:577-581

Logendra L S, T J Gianfanga, D R Specca, H W Janes (2001) Greenhouse tomato limited cluster production systems: crop management practices affect yield. HortScience 36:893-896.

Manjares M M J, R Ferrera-Cerrato, M C González-Chávez (1999) Efecto de la vermicomposta y la micorriza arbuscular en el desarrollo y tasa fotosintética de chile serrano. Terra 17:9-15.

Márquez H C, P Cano R (2004) Producción de tomate orgánico bajo invernadero. In: 2do. Simposium Internacional de Producción de Cultivos en Invernadero. C Leal Ch, J A G Garza (eds). 20 y 21 de mayo. Monterrey, NL, Fundación UANL y Facultad de Agronomía, UANL. pp:1-11.

Márquez H C, P Cano R, Y I Chew M, A Moreno R, N Rodríguez D (2006) Sustratos en la producción de tomate cherry bajo invernadero. Rev. Chapingo S. Hort. 12:183-189.

Milles J A, M M Peet (2002) Maintaining nutrient balances in systems utilizing soluble organic fertilizers. Horticultural Science Department. North Carolina State University. Organic Farming Research Foundation Project Report. Disponible en http://www.ofrf.org/publications/Grant\%20reports/00.23.08. Peet.Spr00.IB12.pdf. (consulta 15 de noviembre del 2005).

Moreno-Resendez A, T Zarate, P M T Valdés L (2005) Desarrollo de tomate en sustrato de vermicomposta/arena bajo condiciones de invernadero. Agric. Téc. (Chile) 65:27-34.

Muro J, E Irigoyen I, M C Salas, M Ureresterazu (2003) Evaluación de un nuevo sustrato de cultivo procedente de residuos madereros en el cultivo hidropónico de tomate en la Cornisa Cantábrica. In: Sustratos-Fertilización Actas de Horticulturae $\mathrm{N}^{\circ}$
39. X Congreso Nacional de Ciencias Hortícolas. Pontevedra. Disponible en: http://www.sech.info/pdfs/actas/ acta39/39 215.PDF. (consulta 15 de diciembre del 2005).

Ndegwa P M, S A Thompson, K C Dass (2000) Effects of stocking density and feeding rate on vermicomposting of biosolids. Bioresource Technol. 71:5-12.

NMX-FF-031-1997-SCFI (1997) Productos alimenticios no industrializados para consumo humano - hortalizas frescas - tomate (Lycopersicun esculentum Mill.)- Especificaciones. SAGARPA. Disponible en: http://www.sagarpa.gob.mx/subagri/ normas/agricolas/catalogos/agricolas/hortalizas/jitomate/nmx jito mate.pdf . (consulta 5 de febrero del 2006).

NRCS. 1999. Agricultural Waste Management Field Handbook. Natural Resource Conservation Service. USDA.

Ortega F S, L Ben-Hur, H Valdez, H Paillan (2003) Efecto de cuatro láminas de agua sobre el rendimiento y calidad de tomate de invernadero producido en primavera verano. Agric. Téc. (Chile) 63:394-402.

Raviv M O, S Medina, A Krasnovsky, H Ziadna (2004) Organic matter and nitrogen conservation in manure compost of organic agriculture. Compost Sci. Util. 12:6-10.

Raviv M O, J Katan, Y Hadar, A YogevS Medina, A Krasnovsky, H Ziadna (2005) High- Nitrogen compost as a médium for organic container grow crops. Biores. Technol. 96:419-427.

Rippy F M J, M M Peet, F J Louws, P V Nelson, D B Orr, K A Sorensen (2004) Plant development and harvest yield of greenhouse tomatoes in six organic growing systems. HortSience 39:223-229.

Ruiz F J F (1998) La agricultura convencional fuente de contaminación del suelo y agua. In: Memorias del III Foro Nacional sobre Agricultura Orgánica. Guadalajara, Jal. Méx. 5 al 7 de noviembre. Consejo Estatal de Promoción Económica del Gobierno del Estado de Jalisco, Universidad de Guadalajara y Consejo Nacional Regulador de Agricultura Orgánica. pp:2930.

SAS (1998) Statistical Analysis System (SAS) versión 6.12. Cary, N.C., USA.

Sloan A E (2002) The natural and organic foods markeplace. Food Technol. 56:27-37.

Tuzel Y, B Yagmur, M Gumus (2003) Organic tomato production under greenhouse conditions. Acta Hort. 614:775-780.

Urrestarazu M, M C Salas, M I Padilla, J Moreno, M A Elorrieta, G A Carrasco (2001) Evaluation of different composts from horticultural crop residues and their uses in greenhouse soilless cropping. Acta Hort. 549:147-152.

Willer H, M Yussefi (2000) Organic agriculture worldwide. IFOAM. Disponible en: http://www.soel.de/inhalte/publikationen/ s_74_02.pdf. (consulta 15 de febrero del 2006).

Zaidan O (1997) El cultivo de tomate de mesa en terreno abierto. In: Curso Internacional de Producción de Hortalizas en Diferentes Condiciones Ambientales. Recopilación de artículos sobre: producción de tomate. O Zidan, R Natan MASHAV (eds). Ministerio de Relaciones Exteriores Centro de Cooperación Internacional. CINDACO. Shefayim, Israel. $18 \mathrm{p}$. 